\title{
Imperfect Quality Certification in Lemons Markets
}

\author{
Birendra K. Mishra', Ashutosh Prasad', Vijay Mahajan² \\ ${ }^{1}$ School of Business, University of California Riverside, Riverside, USA \\ ${ }^{2}$ Department of Marketing, McCombs School of Business, University of Texas at Austin, Austin, USA \\ Email: barry.mishra@ucr.edu
}

How to cite this paper: Mishra, B. K. Prasad, A., \& Mahajan, V. (2020). Imperfect Quality Certification in Lemons Markets. Theoretical Economics Letters, 10, 1260-1275.

https://doi.org/10.4236/tel.2020.106077

Received: September 22, 2020

Accepted: December 12, 2020

Published: December 15, 2020

Copyright (c) 2020 by author(s) and Scientific Research Publishing Inc. This work is licensed under the Creative Commons Attribution International License (CC BY 4.0).

http://creativecommons.org/licenses/by/4.0/

\begin{abstract}
In markets with information asymmetry, the seller of a high-quality product is unable to credibly communicate its quality to buyers and is forced to price like an average quality seller. This is a disincentive to provide quality and high-quality sellers may exit the market. Of several methods to reduce information asymmetry, we provide an analytical study of certification or grading of quality levels by infomediaries. In the equilibrium of a quality reporting game, we find that certification reduces, but does not eliminate, the problems of information asymmetry. There exists a threshold, determined by the accuracy of the certification process, below which customers should believe quality reports, but disbelieve reports above it. We further examine a two-category scheme of high/low quality certification and discuss the design of certification grades using an entropy approach.
\end{abstract}

\section{Keywords}

Product Quality, Certification, Report Verification, Grading, Information Asymmetry

\section{Introduction}

There are many markets in which sellers know the quality of their products but potential buyers do not, unless they buy the product and experience it. Products like these are called experience goods (Nelson, 1974). It is known that the information asymmetry between buyers and sellers can create inefficient markets and market failure. Applications of asymmetric information extend to energy trading (e.g., Li, Chen, \& Nan, 2018) and supply chain management (e.g., Chen et al., 2017). Examples of markets with asymmetric information about quality in particular include both new and less well-known products, used products, and sales 
through internet auctions. Methods to resolve information asymmetry include signaling (Spence, 1974) with branding, price or advertising (Tirole, 1988), scarcity (Stock \& Balachander, 2005), and warranties (e.g., Lutz \& Padmanabhan, 1995). The approach that we will follow is certification which involves the use of third parties. As Akerlof (1970) noted, there is a role for a third-party intermediary, or "infomediary", to provide an objective assessment of the seller's quality to the buyer. Such intermediaries provide a valuable market-making function. In this paper we look at an imperfect quality certification game between the buyer, seller and third party.

We briefly overview several scenarios including certification that involve a third party for quality assurance. In the first scenario, the seller hires a third party to verify its quality claim. Examples are in the literature on quality certification (Heinkel, 1981; Leland, 1979), the auditing literature in accounting (e.g. Newman, Rhoades, \& Smith, 1996), as well as other examples. The seller reports its quality and provides a product sample or other requested information to the third party and asks it to certify its report. A second scenario is where the third party measures the information directly and sells this information to buyers. Papers by Iyer \& Soberman (2000), Sarvary (2002), and Sarvary \& Parker (1997) among others have considered this situation. As a solution, it is equivalent to the buyer hiring a third party. In this scenario the buyer may specify exactly what information is needed, such as specific marketing research tasks. Finally, a third scenario can be observed where the third party is independently sponsored, assesses the quality of the product and makes this information freely available to buyers. Examples are opinion websites on the Internet, consumer reports on many household items, movie critics for movies (e.g., Baranchuk \& Prasad, 2017; Eliashberg \& Shugan, 1997), restaurant guides etc.

In this paper, we focus on the scenario, termed report verification, where the seller hires the third party to certify its quality claims. Our finding is that while this approach is beneficial, it does not fully resolve the information asymmetry problem under some simple conditions. We identify situations where it works and where it does not. We refer hereafter to the third party as the certifier and to the report verification process as certification. The contribution of this paper is to examine for the first time product certification in marketing and show how imperfect certification can reduce information asymmetry while not eliminating it completely by characterizing the equilibrium of a reporting game. We examine cases where quality is a continuum or where it takes discrete values. We also examine the design of a grading scale of the certifier, chosen to maximally reduce the information asymmetry concern.

Product certification is a common practice. Quality seals include Woolmark, which identifies pure wool products from those that may or may not be; the Conformitè Europëenne (CE) mark to show that the product meets the safety and environmental standards of the EU; The American Dental Association's (ADA) seal of acceptance on dental products; British Standards Institute (BSI) 
mark for products that meet safety standards, such as pressure cookers and helmets; The Japanese grading system uses a 12-point scale for Wagyu meat. Even within a supply chain, audits and certification are required; consider the problem for Wal-Mart in verifying whether its fish suppliers from around the world use sustainable sources. The monitoring and auditing task is done by the Marine Stewardship Council. In services, education degrees which divide labor into distinct categories; licensing exams for various professionals; and certifications of skills such as in the software market are quite common.

In the above examples, certification served to categorize, or grade, sellers into a few discrete categories. The certified category established a minimum quality standard that leads to a reduction in information asymmetry and, in many cases, an increase in welfare (Leland, 1979). There are other cases, however, where certification uses a finer grading scale which can be treated as approximately continuous. Some examples are:

- The circulation figures of magazines and newspapers, audience of TV and radio, and website traffic counts are private information for the media but determine the quality of the media for advertisers. The media manager's quality report is finely graded with respect to circulation and demographics, and the report is certified by a third party such as the Audit Bureau of Circulations $(\mathrm{ABC})$ for newspapers and magazines, and IPRO for the Internet. However, for television and radio, the predominant solution to information asymmetry is for the advertiser to buy information from third parties Nielsen and Arbitron for TV and radio respectively, as opposed to the seller hiring the third party. It can be quite difficult for the certifier to verify the numbers reported by the media provider. For example, newspapers require physical verification of circulation and demographic figures. The auditor checks the printed output, subscription lists and physical delivery. A description is provided by a commentator who is responding to the trend to use general accounting firms rather than $\mathrm{ABC}$ auditors: "Will their three-piece suits go digging through dumpsters for discarded newspapers being counted as paid? Or knock on a door in ghetto areas to verify subscribers? Will they define 'paid' at a minimum of $50 \%$ of basic prices? Or will it be $25 \%$ ?" (Schiller, 1998). The answers are important because a publisher can always temporarily enhance circulation through freebies, discounts and special events but they should not claim this circulation figure regularly for setting advertising rates.

- The valuation for firms seeking to attract investment can also be stated precisely. In this case, the firm's management has private information about the true value of the firm and makes a report which is certified by an auditor. Certification by independent accountants is mandatory for all publicly traded firms in the US. Potential investors receive the audited report. It is possible to give several examples that show that it is not possible for the auditor to perfectly detect misreports. In a well-known case, Enron, one of the ten largest companies in the United States went into bankruptcy in 2002 resulting in its 
many investors sustaining billions of dollars in losses. Although auditing should have revealed problems with the firm's finances years in advance, it failed to do so. The auditor Anderson, one of the five largest auditors globally, also failed to detect problems at several other firms.

- Product specifications can be quite precise and companies such as Lloyd's Register provide services to verify manufacturer's product specification claims in many different industries. The Wikipedia entry on "product certification" provides still more examples.

These examples show that certification is widely used in many markets. We noted that the certified quality can be discrete or continuous. We also noted that the certification process does not imply a perfect detection of misreports. Flaws are inherent in any process. For example, Mann (2002) describes software glitches that resulted in the destruction of a Mars mission at NASA, an organization renowned for its stringent quality control. We use these insights in the next section.

\section{Model}

A seller sells a product of quality known only to itself to a buyer. Quality, denoted by $Q$, is a random variable with distribution $F(\cdot)$, continuous density $f(\cdot)$, and bounded support $[0, \bar{t}] \subseteq \mathbb{R}^{+}$. Thus, the seller obtains a realization $q$ of the random variable whereas, for the buyer, the seller's expected quality $e x$ ante is $\mu=\int_{0}^{\bar{t}} x f(x) \mathrm{d} x$. The seller's revenue is equated to the expectation of the buyer's belief about its quality.

The seller can make a report about its quality, denoted $R$. If $R=q$ the seller is being truthful otherwise it is misreporting. By itself, a report $R>\mu$ to the buyer will not be credible. Buyers, lacking verification about the actual quality, will be willing to pay only $\mu$. Thus, the task of a high quality seller is to separate itself out from hypothetical low quality sellers.

So far, this describes a lemons market (Akerlof, 1970). We now extend it to include the certification process. Stated below are the several additional features:

- The seller hires the certifier for a fixed fee. We set it to zero, or assume the certification is mandatory, so that the fee itself is not a factor for information asymmetry to persist.

- The seller makes its report $R$ to the certifier and provides supporting evidence to verify or correct the report made. The certifier either accepts, or rejects-and-corrects, the seller's report, and then releases a certified report $\hat{R}$ to buyers. If the seller's report is accepted then $\hat{R}=R$. If it is rejected, the ensuing detailed examination reveals the true quality and $\hat{R}=q$. This commonly used type of certification, called report verification, is used by auditors (Newman, Rhoades, \& Smith, 1996) and taxation authorities (Reinganum \& Wilde, 1986) among others.

- The realization of quality $q$ is known only to the seller. The seller's report $R$, its supporting evidence, and whether or not the report was accepted or re- 
jected, are known only to the seller and the certifier. All else is common knowledge.

- There is no penalty to the seller for misreporting. In many practical cases no punishment is done because the misreport is detected and corrected in private between the seller and certifier, or it is difficult to prove deliberate intent, and since the certifier is hired by the seller, it can withdraw its services and be replaced.

- The certification process detects misreports with a constant probability $\delta$. If $\delta=1$, the certified report is accurate and the information asymmetry problem is resolved. In practice it is unlikely to be the case that detection is perfect, so we assume that the certifier detects and corrects a misreport with probability $\delta \in[0,1)$ and conversely with probability $1-\delta$ it erroneously certifies the misreport provided by the seller.

The seller maximizes its payoff with respect to its report, i.e.,

$$
\max _{R} E[b(q \mid \hat{R})],
$$

where $E[\cdot]$ is the expectation operator and $b(q \mid \hat{R})$ is the buyer's belief about the quality of the product conditional on observing $\hat{R}$ (note that the buyer does not observe $R$ ).

\section{Analysis and Discussion}

The Perfect Bayesian equilibrium of the game is the reporting strategy $R(q)$ of the seller and posterior beliefs $b(q \mid \hat{R})$ of the buyer, such that the seller's strategy is optimal given the beliefs and the beliefs are obtained from equilibrium strategies and observed actions using Bayes' rule wherever possible. While there may be multiple equilibria of the game, it is most relevant to look at the equilibrium with maximum reduction in information asymmetry for a given detection probability. This is in Proposition 1 (all proofs are in the appendix).

Proposition 1: The Perfect Bayesian Equilibrium of the game, which has maximum reduction of information asymmetry, is characterized as follows:

1) There exists a unique threshold quality $t^{*}$ given by the solution to $t^{*}=\mu+\delta \int_{0}^{t^{*}} F(x) \mathrm{d} x$. It has the properties that $t^{*} \in[\mu, \bar{t}]$ and $\frac{\partial t^{*}}{\partial \delta}>0$.

2) The seller reports $R$, independent of $q$, using a mixed strategy distribution with positive probability everywhere on support $\left[t^{*}, \bar{t}\right]$.

3) Buyers' beliefs are $b\left(q \mid \hat{R} \in\left[0, t^{*}\right)\right)=\hat{R}$ with probability 1 and $b\left(q \mid \hat{R} \in\left[t^{*}, \bar{t}\right]\right)$ is $q \in\left[t^{*}, \bar{t}\right]$ with probability $\frac{1-F\left(t^{*}\right)}{1-\delta F\left(t^{*}\right)}$ and $q \in\left[0, t^{*}\right]$ with probability $\frac{(1-\delta) F\left(t^{*}\right)}{1-\delta F\left(t^{*}\right)}$. Therefore, $E\left[b\left(q \mid \hat{R} \in\left[0, t^{*}\right)\right)\right]=\hat{R} \quad$ and $E\left[b\left(q \mid \hat{R} \in\left[t^{*}, \bar{t}\right]\right)\right]=t^{*}$. 
4) Thus, expected payoff to sellers of type $q \in\left[0, t^{*}\right)$ is $\delta q+(1-\delta) t^{*}$ and to types $q \in\left[t^{*}, \bar{t}\right]$ is simply $t^{*}$.

5) For $F(x)$ uniform on $[0, \bar{t}]$ we find that $t^{*}=\bar{t} /(1+\sqrt{1-\delta})$.

Thus, certification does achieve reduction in information asymmetry by establishing a threshold such that buyers completely believe reports of less-than-threshold quality. The threshold value $t^{*}$ increases, and the information asymmetry decreases, with the detection probability $\delta$. Indeed, when $\delta=0$, we get $t^{*}=\mu$ as in the case with no intermediary, and when detection is perfect, i.e., $\delta \rightarrow 1$, the information asymmetry is completely resolved because $t^{*} \rightarrow \bar{t}$, which is intuitive.

The implications of the equilibrium properties in Proposition 1 are serious if sellers at the high end exit the market as opposed to charging below their actual quality. Proposition 1 states that types higher than $t^{*}$ will be evaluated below their actual quality and therefore have little incentive to trade. Thus, the main problem of "lemons" markets, that high quality sellers are unable to credibly communicate their quality to buyers, persists despite intermediation if by an imperfect certifier. This also shows that a higher $\delta$ is beneficial. In a study of baseball cards, Jamal \& Sunder (2011) find that the market values the service of strict graders more than lenient graders.

To increase the precision of detection, a better technology or more effort can be applied subject to some tradeoff with costs. Redundancy can be useful, in the sense of having more than one examiner or inspection, but also costly. Suppose that there are $v$ independent examiners, indexed by $i$, with detection probabilities $\delta_{i}$. Then from probability theory, their combined detection probability will be equal to $1-\prod_{i=1}^{v}\left(1-\delta_{i}\right)$. Thus, if there are two examiners who each have a detection probability of 0.7 , their combined detection probability increases to 0.91 .

This paper shows certification to be less beneficial than in some other papers. For example, Viscusi (1978) showed that the certification can result in a full information outcome even when only the highest quality sellers would initially want to pay the certification fee to reveal their quality. This is because once these types have their quality revealed, buyers' valuations for the remaining sellers is lowered, giving an incentive for the highest unrevealed types to have their quality certified. The entire industry may thus, in sequence, have their quality certified. In the present case, the certification fee is unimportant, since the market can unravel even if the fee is zero. The contrasting result is caused by other different assumptions under which the results are obtained. The difference is that here the detection process is imperfect, whereas the certification process assumed in Viscusi (1978) is perfect. Secondly, we assume that misreporting is unpunished. Heinkel (1981) considers an imperfect detection technology but examines the issue of a penalty on the seller if the buyer finds the quality to be suboptimal after the sale and initiates legal arguments of product liability or false advertising, and finds that penalty results in an improvement of welfare. 


\section{The Discrete Case}

For further insight, we will discuss the use of discrete grading scales used by certifiers and of which several examples were given in the introduction. Discrete grades have the advantage that customers are able to process and act on their information more easily and the grader also faces an easier task which may explain their prevalence. We find linkages in grading design between the results here and those in Proposition 1.

We consider the case where a seller will be classified into one of two possible quality grades, LOW or HIGH. The prior probabilities are $\pi(x)$ and $1-\pi(x)$ respectively and $x$ is the cutoff. The equilibrium strategy is for the seller to always report its quality as High, since the alternative report, for this case, is clearly dominated. The certifier detects and corrects the misreports with probability $\delta$. The process is depicted in Figure 1 where the horizontal bar is the quality line from $[0, \bar{t}]$ and on it $x$ is the cutoff between the two grades.

When the buyers see a certified report claiming Low quality, they comprehend that the report has been detected and corrected by the certification process, and accept it as true. However, if buyers see a certified report claiming High quality, they are unsure whether the seller actually has High quality, or whether this is an undetected misreport. The posterior probability (belief) that the seller is actually High quality upon observing a certified report of High quality is,

$$
\operatorname{Pr}(q=\operatorname{High} \mid \hat{R}=\text { High })=\frac{1-\pi(x)}{1-\delta \pi(x)},
$$

Suppose that $\pi(x)=0.9$ and $\delta=0.99$. Then the posterior probability is close to 1 , implying near-certainty on the part of the buyer.

We summarize the results of this problem into Proposition 2.

Proposition 2: The Perfect Bayesian Equilibrium of the game with discrete High/Low grades is characterized as follows:

1) The seller reports $R=$ High independent of $q$.
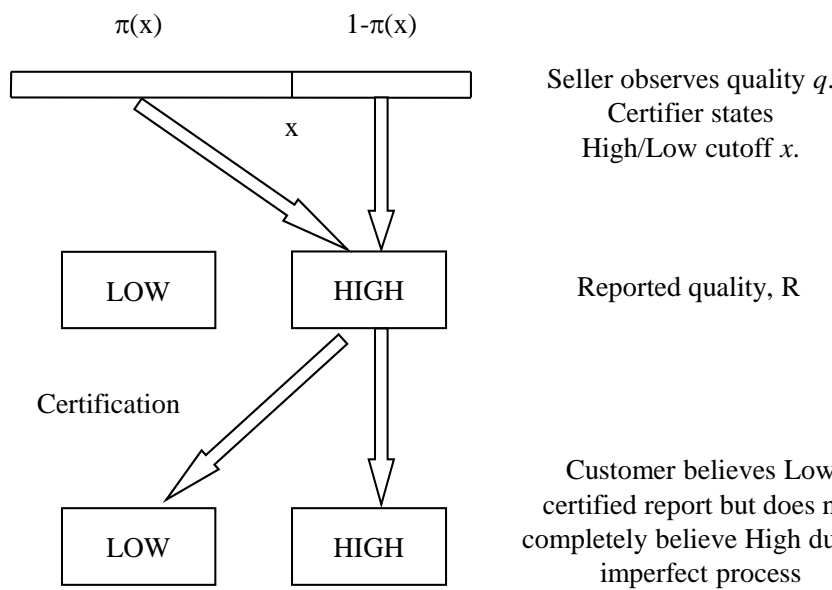

Reported quality, $\mathrm{R}$

$$
\begin{aligned}
& \text { Customer believes Low } \\
& \text { certified report but does not } \\
& \text { completely believe High due to } \\
& \text { imperfect process }
\end{aligned}
$$

Figure 1. Reporting process in discrete case. 
2) Buyers' beliefs are $b(q \mid \hat{R}=$ Low $)=$ Low with probability 1 , while $b(q \mid \hat{R}=$ High $)$ is $q=$ High with probability $\frac{1-\pi(x)}{1-\delta \pi(x)}$ and $q=$ Low with probability $\frac{(1-\delta) \pi(x)}{1-\delta \pi(x)}$.

3) The value of $x$ that maximizes the informativeness of the grades is given by $\pi(x)=1 / 2 \delta$ for $\delta \geq 0.5$ and $\pi(x)=1$ for $\delta<0.5$. Thus, for $\delta \rightarrow 1$, $x \rightarrow \pi^{-1}(1 / 2)$, the median quality.

Upon seeing a certified report claiming High quality, a higher than $1-\pi(x)$ belief is assigned that the seller is High quality, and this increases with the detection probability $\delta$ as one would intuitively expect. Values of $\delta$ less than 1 do not allow a full resolution of the information asymmetry. In particular, when $\delta=0$ implying that all misreports will escape detection, the report is uninformative and the buyers' prior remains unchanged. We can appreciate that the certification process has to have a reasonably large detection probability to be useful. Furthermore, observe that "reasonably large" depends on the magnitude of $\pi(x)$. For a low probability event, such as a seller claiming to be the best in its class out of many sellers, the prior belief $1-\pi(x)$ is quite small. This leads to the posterior belief upon observing the certified report to also be small. Thus, consider the example where the prior for the quality being High is $1-\pi(x)=0.01$ and $\delta=0.99$. The posterior is only about 0.5 . For the same detection probability $\delta=0.99$, there is much less certainty upon seeing a certified report of High quality when the prior $1-\pi(x)=0.01$ than when it is $1-\pi(x)=0.1$.

In the limit, with the expected probability of High quality sellers is infinitesimally small, the posterior belief can be written as,

$$
\lim _{\pi(x) \rightarrow 1} \frac{1-\pi(x)}{1-\delta \pi(x)}=0,
$$

i.e., the prior and posterior are both zero. In other words, if there are very few High quality sellers, a report of High quality carries little value regardless of the detection function, as long as the detection is not perfect. As the saying goes, exceptional claims require exceptional evidence, and this cannot be provided if the detection process is imperfect.

The results in Proposition 2(a) and 2(b) are analogous to what Proposition 1 states. Under Proposition 1, every seller would report itself somewhere in the quality range $\left[t^{*}, \bar{t}\right]$. It will not always be the highest possible quality $\bar{t}$ because with a continuous distribution of quality types, the probability of having exactly a certain quality level is a probability zero event. Consequently, observing a certified quality report $\bar{t}$ has no informational value to buyers that quality is $\bar{t}$, but it does update their priors that quality is in the range $\left[t^{*}, \bar{t}\right]$. This shows that the market suffers from a similar problem as in Akerlof (1970) as the high- 
est types are unable to completely separate themselves out even with certification. Thus, this can lead to a market failure even under certification.

We next turn to Proposition 2(c) which is obtained from an analysis maximizing entropy, equivalent to maximizing the information from the certification grades. As an aside, we note that maximizing information from the grading scale is better than maximizing the proportion of products correctly classified based on posterior beliefs of consumers.

From the matrix shown in Table 1 , the problem is,

$$
\max _{\pi} \pi\left[\delta+\frac{(1-\delta)^{2} \pi}{1-\delta \pi}\right]+(1-\pi) \frac{1-\pi}{1-\delta \pi} .
$$

After some algebra, this simplifies to,

$$
\max _{\pi} \frac{1+2 \pi^{2}(1-\delta)-\pi(2-\delta)}{1-\delta \pi} .
$$

This function is convex, however, with maximum occurring at the end points $\pi=0$ or $\pi=1$. In either case, the problem is reduced to having a single category where indeed there is no possibility of incorrect classification. Interestingly, the value of $\pi$ that minimizes this function is given by the cutoff corresponding to $t^{*}$ in the continuous case. The first order condition for the interior minimum, after simplifications, reduces to $\delta \pi^{2}-2 \pi+1=0$, from which the solution is:

$$
\pi=\frac{1-\sqrt{1-\delta}}{\delta}=\frac{1}{1+\sqrt{1-\delta}} .
$$

This shows that maximizing the belief about correct classification is not the same as having the most informative scale.

The use of the discrete grade categorization is convenient and used in many cases, but there is loss of information associated with discretizing an underlying continuous quality scale down to two categories. The problem is to design the categories so that maximum information is retained. The literature in Chen et al. (2015) and some other scale design papers examine this through a behavioral study whereas here, we take an analytical approach. In contrast to Baranchuk and Prasad (2017) where the third party knows the exact product quality without error, here the imperfect certification process plays a key role.

A measure of information is entropy. Let $\left\{A_{i}\right\}_{i=1}^{N}$ be a partition (a set of exhaustive and mutually exclusive events) of an underlying experiment, with associated probabilities $\left\{p_{i}\right\}_{i=1}^{N}$. That is, $p_{i}$ is the probability of event $A_{i}$. Then a measure of the uncertainty of the partition is the entropy, defined as $-\sum_{i=1}^{N} p_{i} \log p_{i} \quad$ (Papoulis 1984). Following the discussion in the appendix, the events HIGH and LOW occur with probabilities $1-\delta \pi(x)$ and $\delta \pi(x)$, respectively. A design that always gives a HIGH or LOW irrespective of the actual quality does not provide much information. Some uncertainty (information) is required, and Proposition 2(c) provides the optimal cutoff. 
Table 1. Classification matrix.

\begin{tabular}{ccc}
\hline & $\begin{array}{c}\text { Posterior probability of being } \\
\text { believed LOW }\end{array}$ & $\begin{array}{c}\text { Posterior probability of being } \\
\text { believed HIGH }\end{array}$ \\
\hline $\begin{array}{c}\text { Product Quality LOW } \\
\text { (proportion } \pi \text { ) }\end{array}$ & $\delta+\frac{(1-\delta)^{2} \pi}{1-\delta \pi}$ & $\frac{(1-\delta)(1-\pi)}{1-\delta \pi}$ \\
$\begin{array}{c}\text { Product Quality HIGH } \\
\text { (proportion } 1-\pi)\end{array}$ & $\frac{(1-\delta) \pi}{1-\delta \pi}$ & $\frac{1-\pi}{1-\delta \pi}$ \\
\hline
\end{tabular}

Figure 2 shows some optimal cutoffs from using the formula in Proposition 2 (c), given an underlying uniform distribution of quality. Note that the cutoff for a grade of HIGH should become more difficult to achieve as the detection probability decreases.

A finer partition will always increase entropy. This means that the two category grading scale could be improved upon by using more grade categories to make it more informative. Of the examples we cited, baseball card rating, by PSA, Beckett etc. had a high number of grade categories of twenty, i.e., ten and half points being possible. Gold purity is measured in Karats from 1 to 24 . Due to its color and malleability gold is often used for jewelry making and a purity of 14 to 22 karat is used. But certification for gold must be stringently carried out because adulteration is a concern and indeed has been a problem since historical times, e.g., there is the ancient story of Archimedes solving this problem by using buoyancy. Fine grades are used by coin collectors, with grading services provided by firms such as Numismatic Guaranty Corporation (NGC) and Professional Coin Grading Services (PCGS). The grades are shorthand to indicate the appearance of a coin. If one collector tells another about a "Saint Gaudens \$20 Gold Double Eagles MS64" both collectors understand about the appearance quality of the coin, even if one has never seen it, due to its grade MS64. This is from the 70-point Sheldon Scale used for grading coins. From the NGC website, the highest grade is MS70, for a Mint State coin that has no post production imperfections at 5 times magnification.

The tradeoff is that more grades, and in the extreme case the continuous measure examined in the previous section, might be confusing from a practical perspective. Also from a practical perspective, if the grades are too fine relative to the detection probability then one might expect an increased error of misclassification. Fewer grades can increase participation (Harbaugh \& Rasmusen, 2018). In other cases an increase in the number of grade categories can be informative such as when restaurant hygiene grading was changed from pass or fail to letter grades (Jin \& Leslie, 2003). Not only the sales at the restaurants but also food related illnesses decreased. Lizzeri (1999) also considered cases where the certifier might choose to provide a less informative pass/fail or high/low report or something more informative. Also, for grading students in exams or employees for wages, Dubey \& Geanakoplos (2010) find that having more grades motivates the individual to apply more effort. In our case, quality and market participation are exogenous and we adopt an entropy maximizing approach. 


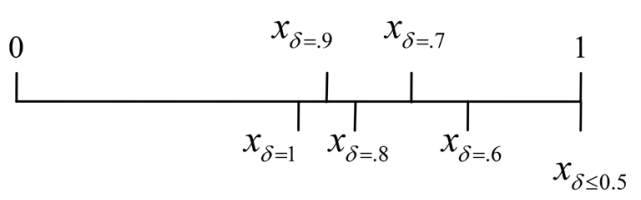

Figure 2. Cutoffs for different values of $\delta$.

\section{Conclusion}

This paper examined the use of an imperfect certifier to resolve the information asymmetry between the buyers and seller. The seller reports its quality and provides supporting evidence to the certifier, who produces a certified report for buyers. We characterized the equilibrium for the case of mandatory certification and no penalty for misreporting. The results show that quality certification reduces but does not completely eliminate the information asymmetry problem. Notably, higher than threshold quality sellers may still exit the market, unraveling the market from the high end similar to Akerlof (1970).

Proposition 1 provides the solution to the game between the firm, the certifier and the customers. The firm would like the customers believe that its quality is as high as possible, but rationally they do not. When the report is certified, Proposition1 finds that the buyers should not completely believe the report when it is larger than a threshold but should believe it if it is below the threshold. The threshold is determined by the detection technology of the certifier. Thus, sellers are not uniformly benefitted. Because information asymmetry is not fully removed given that the detection probability of the certification device is likely to be imperfect, other methods for removing information asymmetry should also be considered.

In Proposition 2 we examined the case where the certification grades are discrete, of the type High/Low or Pass/Fail that is sometimes seen in practice. For example, the use of the Woolmark brand for pure wool products. Even though this is a coarse scale, it provides useful information to customers. As we discussed, it may be less useful when the prior probability of any quality level occurring is low, as may happen with media circulation figures, firm valuation and product specifications. In Proposition 2, we also determined the best design of categories for providing information about the product quality using an entropy-based approach.

Future research should continue to examine and compare solutions to the information asymmetry problem. It may be that for certain situations it is better for the third party to be sponsored by the buyer and in other cases by the seller. This would explain why examples of both are seen in practice, for example, in the reporting of firm valuation, sellers hire the third party, while in the market for investment advice, buyers hire the third party. Once we start examining the incentives of the certifier, it may be possible that the certifier can invest in improving its certification accuracy.

Future research may also take an empirical route to verify our result that cer- 
tification is useful only when it is perfect or when it is used in conjunction with other enforcing mechanisms.

\section{Acknowledgements}

The authors are grateful to Nina Baranchuk and Rami Zwick for their helpful suggestions.

\section{Conflicts of Interest}

The authors declare no conflicts of interest regarding the publication of this paper.

\section{References}

Akerlof, G. A. (1970). The Market for Lemons: Quality Uncertainty and the Market Mechanism. Quarterly Journal of Economics, 84, 488-500.

https://doi.org/10.2307/1879431

Baranchuk, N., \& Prasad, A. (2017). Making the Grade: Product Quality Reporting by Infomediaries. University of Texas at Dallas Working Paper.

Chen, L., Peng, J., Liu, Z., \& Zhao, R. (2017). Pricing and Effort Decisions for a Supply Chain with Uncertain Information. International Journal of Production Research, 55, 264-284. https://doi.org/10.1080/00207543.2016.1204475

Chen, X., Yu, H., \& Yu, F. (2015). What Is the Optimal Number of Response Alternatives for Rating Scales? From an Information Processing Perspective. Journal of Marketing Analytics, 3, 69-78. https://doi.org/10.1057/jma.2015.4

Dubey, P., \& Geanakoplos, J. (2010). Grading Exams: 100, 99, 98... or A, B, C? Games and Economic Behavior, 69, 72-94. https://doi.org/10.1016/j.geb.2010.02.001

Eliashberg, J., \& Shugan, S. M. (1997). Film Critics: Influencers or Predictors? Journal of Marketing, 61, 68-78. https://doi.org/10.1177/002224299706100205

Harbaugh, R., \& Rasmusen, E. (2018). Coarse Grades: Informing the Public by Withholding Information. American Economic Journal: Microeconomics, 10, 210-235. https://doi.org/10.1257/mic.20130078

Heinkel, R. (1981). Uncertain Product Quality: The Market for Lemons with an Imperfect Testing Technology. Bell Journal of Economics, 12, 625-636. https://doi.org/10.2307/3003577

Iyer, G., \& Soberman, D. (2000). Markets for Product Modification Information. Marketing Science, 19, 203-226. https://doi.org/10.1287/mksc.19.3.203.11801

Jamal, K., \& Sunder, S. (2011). Is Mandated Independence Necessary for Audit Quality? Accounting, Organizations and Society, 36, 284-292.

https://doi.org/10.1016/j.aos.2011.03.001

Jin, G., \& Leslie, P. (2003). The Effects of Information on Product Quality: Evidence from Restaurant Hygiene Grade Cards. Quarterly Journal of Economics, 11, 409-451. https://doi.org/10.1162/003355303321675428

Leland, H. E. (1979). Quacks, Lemons and Licensing: A Theory of Minimum Quality Standards. Journal of Political Economy, 87, 1328-1346. https://doi.org/10.1086/260838

Li, Z., Chen, L., \& Nan, G. (2018). Small-Scale Renewable Energy Source Trading: A Contract Theory Approach. IEEE Transactions on Industrial Informatics, 14, 1491-1500. https://doi.org/10.1109/TII.2017.2776241 
Lizzeri, A. (1999). Information Revelation and Certification Intermediaries. Rand Journal of Economics, 30, 214-231. https://doi.org/10.2307/2556078

Lutz, N., \& Padmanabhan, V. (1995). Why Do We Observe Minimal Warranties? Marketing Science, 14, 417-441. https://doi.org/10.1287/mksc.14.4.417

Mann, C. C. (2002). Why Software Is So Bad. MIT Technology Review, July. https://www.technologyreview.com/2002/07/01/40875/why-software-is-so-bad

Nelson, P. (1974). Advertising as Information. Journal of Political Economy, 82, 729-754. https://doi.org/10.1086/260231

Newman, P., Rhoades, S., \& Smith, R. (1996). Allocating Audit Resources to Detect Fraud. Review of Accounting Studies, 1, 161-182. https://doi.org/10.1007/BF02918230

Papoulis, A. (1984). Probability, Random Variables, and Stochastic Processes (2nd ed.). New York: McGraw-Hill.

Reinganum, J., \& Wilde, L. (1986). Equilibrium Verification and Reporting Policies in a Model of Tax Compliance. International Economic Review, 27, 739-760. https://doi.org/10.2307/2526692

Sarvary, M. (2002). Temporal Differentiation and the Market for Second Opinions. Journal of Marketing Research, 39, 129-136. https://doi.org/10.1509/jmkr.39.1.129.18933

Sarvary, M., \& Parker, P. M. (1997). Marketing Information: A Competitive Analysis. Marketing Science, 16, 24-38. https://doi.org/10.1287/mksc.16.1.24

Schiller, J. (1998). A Defense of the Audit Bureau Auditors. Editor \& Publisher, 131, 30.

Spence, M. (1974). Market Signaling. Cambridge, MA: Harvard University Press.

Stock, A., \& Balachander, S. (2005). The Making of a "Hot Product": A Signaling Explanation of Marketers' Scarcity Strategy. Management Science, 51, 1181-1192. https://doi.org/10.1287/mnsc.1050.0381

Tirole, J. (1988). The Theory of Industrial Organization. Cambridge, MA: MIT Press.

Viscusi, W. K. (1978). A Note on "Lemons" Markets with Quality Certification. Bell Journal of Economics, 9, 277-279. https://doi.org/10.2307/3003627 


\section{Appendix}

\section{Proof of Proposition 1}

The seller's objective is met by a reporting strategy $R(q)$ that mixes over all highest expected payoff points. Taking the beliefs (in Proposition 1) as given, the seller gets, if undetected, a maximum near $t^{*}$ from reporting in $\left[0, t^{*}\right)$ and $\lambda$ (derived below) from reporting in $\left[t^{*}, \bar{t}\right]$. Provided $\lambda \geq t^{*}$, the seller reports in the range $\left[t^{*}, \bar{t}\right]$. Any mixed strategy distribution on $\left[t^{*}, \bar{t}\right]$ is consistent with the beliefs if it has positive probability density everywhere on $\left[t^{*}, \bar{t}\right]$. (Else, an $\hat{R}$ in the gap would imply detection and correction, and be fully believed, making the belief that all $\hat{R}$ in $\left[t^{*}, \bar{t}\right]$ have the same payoff inconsistent with the reporting strategy.)

Taking the seller's reporting strategy as given, the buyer observes $\hat{R}$ in $\left[0, t^{*}\right)$ only if a misreport is corrected, and (by Bayes' rule) it is believed. If $\hat{R}$ is in $\left[t^{*}, \bar{t}\right]$, the buyer applies Bayes' rule to get:

$$
\operatorname{Pr}\left\{q \in\left[t^{*}, \bar{t}\right] \mid \hat{R} \in\left[t^{*}, \bar{t}\right]\right\}=\frac{1-F\left(t^{*}\right)}{1-\delta F\left(t^{*}\right)} .
$$

Beyond this updating, $\hat{R}$ in the range $\left[t^{*}, \bar{t}\right]$ is uninformative, i.e., different $\hat{R}$ give equal payoff to the seller. Suppose not, i.e., let $\lambda$ be the payoff at $\hat{R}=t^{*}$ and: 1) Let $\tilde{t} \in\left(t^{*}, \bar{t}\right]$ be the smallest $\hat{R}$ for which (beliefs are such that) payoff is higher than $\lambda$. But then $t^{*}$ would be $\tilde{t}$, i.e., there will be no $R<\tilde{t}$ and so any $\hat{R}<\tilde{t}$ can only come from certification correction and must be believed. This contradicts $\tilde{t} \in\left(t^{*}, \bar{t}\right]$. 2) Let $\tilde{t} \in\left(t^{*}, \bar{t}\right)$ be a $\hat{R}$ that gives payoff lower than $\lambda$. Then no seller would report $R=\tilde{t}$ but in that case $\hat{R}=\tilde{t}$ can only come from certification correction and must be believed. Since $\tilde{t}>t^{*}$, this is inconsistent with the assumption that $\hat{R}=\tilde{t}$ has a payoff lower than $\lambda$ if $\lambda=t^{*}$, a fact we show below. Hence, the result is proved by contradiction. Finally, out of equilibrium beliefs are unspecified because any $\hat{R} \in[0, \bar{t}]$ can be observed in equilibrium. Next, we determine $\lambda$ as follows:

$$
\begin{aligned}
\lambda & =\frac{1-F\left(t^{*}\right)}{1-\delta F\left(t^{*}\right)} \times \frac{\int_{t^{*}}^{\bar{t}} x f(x) \mathrm{d} x}{1-F\left(t^{*}\right)}+\left(1-\frac{1-F\left(t^{*}\right)}{1-\delta F\left(t^{*}\right)}\right) \times \frac{\int_{0}^{t^{*}} x f(x) \mathrm{d} x}{F\left(t^{*}\right)} \\
& =\frac{1}{1-\delta F\left(t^{*}\right)}\left(\mu-\delta \int_{0}^{t^{*}} x f(x) \mathrm{d} x\right)
\end{aligned}
$$

The condition $\lambda \geq t^{*}$ can be written as,

$$
\begin{gathered}
\mu-\delta \int_{0}^{t^{*}} x f(x) \mathrm{d} x \geq t^{*}\left(1-\delta F\left(t^{*}\right)\right) \\
\Rightarrow t^{*} \leq \mu+\delta \int_{0}^{t^{*}} F(x) \mathrm{d} x
\end{gathered}
$$

Because all reports below $t^{*}$ are accurate, the maximum reduction of information asymmetry occurs when $t^{*}$ has the highest value. The highest value is thus $t^{*}=\mu+\delta \int_{0}^{t^{*}} F(x) \mathrm{d} x$, which exists in $[0, \bar{t}]$ and is unique because: 1$)$ At 
$\mu$ the LHS $<$ RHS. 2) At $\bar{t}$ the LHS $>$ RHS, because the RHS is $\mu+\delta \int_{0}^{\bar{t}} F(x) \mathrm{d} x<\mu+\int_{0}^{\bar{t}} F(x) \mathrm{d} x=\bar{t}$ while the LHS is $\bar{t}$. As both LHS and RHS are continuous, increasing functions of $t^{*}$, this proves existence. 3) The slope $\frac{\mathrm{d}}{\mathrm{d} t^{*}}\left(t^{*}-\mu-\delta \int_{0}^{t^{*}} F(x) \mathrm{d} x\right)>0$ and hence has only one root. This establishes uniqueness.

Applying the implicit function theorem to $t^{*}-\mu-\delta \int_{0}^{t^{*}} F(x) \mathrm{d} x$ yields $\frac{\partial t^{*}}{\partial \delta}=\frac{1}{1-\delta F\left(t^{*}\right)} \int_{0}^{t^{*}} F(x) \mathrm{d} x>0$.

For $F(x)$ uniform on $[0, \bar{t}], t^{*}$ solves $t^{*}=\frac{\bar{t}}{2}+\delta \int_{0}^{t^{*}} \frac{t}{\bar{t}} \mathrm{~d} t$. This implies $2 \overline{t t}^{*}=\bar{t}^{2}+\delta t^{* 2}$ or $t^{*}=\frac{\bar{t}}{1+\sqrt{1-\delta}} \cdot \cdot$

Proof of Proposition 2

2(a) Reporting HIGH dominates reporting LOW for all types given the beliefs (stated in part $2(\mathrm{~b})$ ). Let $q_{H}$ denote $q=\mathrm{HIGH}$ and $q_{L}$ denote $q=\mathrm{LOW}$, and $q_{H}>q_{L}$ by definition. Then the payoff to a seller of HIGH quality from reporting $\mathrm{HIGH}$ is $\frac{q_{H}(1-\pi)+q_{L}(1-\delta) \pi}{1-\delta \pi}$ and from reporting LOW is $q_{L}$. And $\frac{q_{H}(1-\pi)+q_{L}(1-\delta) \pi}{1-\delta \pi}>\left(q_{L}=\frac{q_{L}(1-\pi)+q_{L}(1-\delta) \pi}{1-\delta \pi}\right)$ proves the result. For the LOW type, the payoff from reporting $\mathrm{HIGH}$ is $\delta q_{L}+(1-\delta) \frac{q_{H}(1-\pi)+q_{L}(1-\delta) \pi}{1-\delta \pi}$ and from reporting LOW is $q_{L}$. Comparing these we see that again that the report of HIGH dominates.

2(b) To derive buyers' beliefs, we apply Bayes' Rule:

$$
\begin{aligned}
& b(q=\mathrm{LOW} \mid \hat{R}=\mathrm{LOW}) \\
& =\frac{\operatorname{Pr}(\hat{R}=\mathrm{LOW} \mid q=\mathrm{LOW}) \times \operatorname{Pr}(q=\mathrm{LOW})}{\operatorname{Pr}(\hat{R}=\mathrm{LOW} \mid q=\mathrm{LOW}) \times \operatorname{Pr}(q=\mathrm{LOW})+\operatorname{Pr}(\hat{R}=\mathrm{LOW} \mid q=\mathrm{HIGH}) \times \operatorname{Pr}(q=\mathrm{HIGH})} \\
& =\frac{\delta \times \pi}{\delta \times \pi+0 \times(1-\pi)}=1
\end{aligned}
$$

And,

$$
\begin{aligned}
& b(q=\mathrm{HIGH} \mid \hat{R}=\mathrm{HIGH}) \\
& =\frac{\operatorname{Pr}(\hat{R}=\mathrm{HIGH} \mid q=\mathrm{HIGH}) \times \operatorname{Pr}(q=\mathrm{HIGH})}{\operatorname{Pr}(\hat{R}=\mathrm{HIGH} \mid q=\mathrm{HIGH}) \times \operatorname{Pr}(q=\mathrm{HIGH})+\operatorname{Pr}(\hat{R}=\mathrm{HIGH} \mid q=\mathrm{LOW}) \times \operatorname{Pr}(q=\mathrm{LOW})} \\
& =\frac{1 \times(1-\pi)}{1 \times(1-\pi)+(1-\delta) \times \pi}=\frac{1-\pi}{1-\delta \pi}
\end{aligned}
$$

And its converse, $b(q=\mathrm{LOW} \mid \hat{R}=\mathrm{HIGH})=1-\frac{1-\pi}{1-\delta \pi}=\frac{(1-\delta) \pi}{1-\delta \pi}$. 
2(c) Entropy is defined as $-\sum_{i=1}^{N} p_{i} \log p_{i}$ where in the present case there are $N=2$ possible events observed by the customer, either HIGH or LOW. The probability of the events are obtained as follows: HIGH will be observed for the $1-\pi(x)$ proportion that is actually HIGH as well as for the $\pi(x)$ LOW quality products that misreport HIGH and with probability $1-\delta$ remain undetected. Thus, the probability is $1-\pi(x)+(1-\delta) \pi(x)$, which can be rewritten as $1-\delta \pi(x)$. The probability of observing LOW is therefore $\delta \pi(x)$.

We select the cutoff $x$ that maximizes entropy. Since $\pi(x)$ is a monotone function of $x$, we maximize entropy with respect to $y=\delta \pi(x)$ :

$$
\max _{y}-y \log (y)-(1-y) \log (1-y) .
$$

The first derivative yields (after returning $y=\delta \pi$ ),

$$
-\log (\delta \pi)+\log (1-\delta \pi) .
$$

Note that for $\delta \pi<0.5$, the slope is positive. Equating the slope to zero, and solving, we get $\pi=1 / 2 \delta$. In case $\delta<0.5$, then $\delta \pi<0.5$ because the value of $\pi$ is at most 1 , and there is a corner solution of $\pi=1$. 